\title{
Genome re-sequencing and reannotation of the Escherichia coli ER2566 strain and transcriptome sequencing under overexpression conditions
}

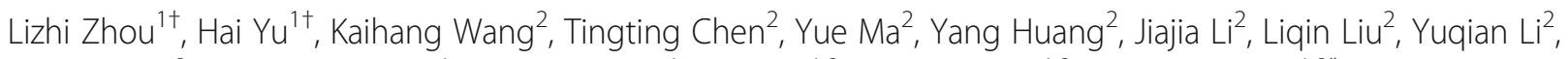
Zhibo Kong ${ }^{2}$, Qingbing Zheng ${ }^{1}$, Yingbin Wang ${ }^{1}$, Ying $\mathrm{Gu}^{1,2}$, Ningshao Xia ${ }^{1,2}$ and Shaowei $\mathrm{Li}^{1,2^{*}}$ (i)

\begin{abstract}
Background: The Escherichia coli ER2566 strain (NC_CP014268.2) was developed as a BL21 (DE3) derivative strain and had been widely used in recombinant protein expression. However, like many other current RefSeq annotations, the annotation of the ER2566 strain was incomplete, with missing gene names and miscellaneous RNAs, as well as uncorrected annotations of some pseudogenes. Here, we performed a systematic reannotation of the ER2566 genome by combining multiple annotation tools with manual revision to provide a comprehensive understanding of the E. coli ER2566 strain, and used high-throughput sequencing to explore how the strain adapted under external pressure.

Results: The reannotation included noteworthy corrections to all protein-coding genes, led to the exclusion of 190 hypothetical genes or pseudogenes, and resulted in the addition of 237 coding sequences and 230 miscellaneous noncoding RNAs and 2 tRNAs. In addition, we further manually examined all 194 pseudogenes in the Ref-seq annotation and directly identified $123(63 \%)$ as coding genes. We then used whole-genome sequencing and highthroughput RNA sequencing to assess mutational adaptations under consecutive subculture or overexpression burden. Whereas no mutations were detected in response to consecutive subculture, overexpression of the human papillomavirus 16 type capsid led to the identification of a mutation (position 1,094,824 within the 3' non-coding region) positioned 19-bp away from the lacl gene in the transcribed RNA, which was not detected at the genomic level by Sanger sequencing.
\end{abstract}

(Continued on next page)

\footnotetext{
* Correspondence: shaowei@xmu.edu.cn

'Lizhi Zhou and Hai Yu contributed equally to this work.

'State Key Laboratory of Molecular Vaccinology and Molecular Diagnostics, School of Public Health, Xiamen University, Xiamen 361102, Fujian, China

${ }^{2}$ National Institute of Diagnostics and Vaccine Development in Infectious Disease, School of Life Sciences, Xiamen University, Xiamen 361102, Fujian, China
}

(c) The Author(s). 2020 Open Access This article is licensed under a Creative Commons Attribution 4.0 International License, which permits use, sharing, adaptation, distribution and reproduction in any medium or format, as long as you give appropriate credit to the original author(s) and the source, provide a link to the Creative Commons licence, and indicate if changes were made. The images or other third party material in this article are included in the article's Creative Commons licence, unless indicated otherwise in a credit line to the material. If material is not included in the article's Creative Commons licence and your intended use is not permitted by statutory regulation or exceeds the permitted use, you will need to obtain permission directly from the copyright holder. To view a copy of this licence, visit http://creativecommons.org/licenses/by/4.0/ The Creative Commons Public Domain Dedication waiver (http://creativecommons.org/publicdomain/zero/1.0/) applies to the data made available in this article, unless otherwise stated in a credit line to the data. 


\begin{abstract}
(Continued from previous page)
Conclusion: The ER2566 strain was used by both the general scientific community and the biotechnology industry. Reannotation of the E. coli ER2566 strain not only improved the RefSeq data but uncovered a key site that might be involved in the transcription and translation of genes encoding the lactose operon repressor. We proposed that our pipeline might offer a universal method for the reannotation of other bacterial genomes with high speed and accuracy. This study might facilitate a better understanding of gene function for the ER2566 strain under external burden and provided more clues to engineer bacteria for biotechnological applications.
\end{abstract}

Keywords: Escherichia coli ER2566, Genome reannotation, Transcriptome sequencing, Engineer bacteria

\section{Background}

The Escherichia coli expression system is one of the most well-characterized classical expression systems for recombinant protein expression in biological science. $E$. coli offers clear advantages over other expression systems, including a clear genetic background, fast breeding, low cost, high cell density cultures, and high protein expression levels [1-3]. Indeed, more than $60 \%$ of recombinant proteins and nearly $30 \%$ of approved recombinant therapeutic proteins are produced using E. coli expression systems [4]. The E. coli ER2566 strain is a common laboratory tool that takes advantage of the expression and growth properties of the B line strain [5]. In 2016, the first complete genome of ER2566 (C2566, NC_CP014268.2) competent cells was sequenced by New England Biolabs and deposited into GenBank [6], with automatic annotation by the NCBI prokaryotic genome annotation pipeline (PGAP) [7].

Along with the rapid development of biological laboratory techniques, there has been a significant advance in sequencing technologies. However, this has not been matched by the production of better sequences; rather, sequencing advancements have led to the deposition of an increased number of "draft" bacterial genomes into public databases [8], which tend to be incomplete and fragmented. In addition, the massive amounts of genomic data generated by next-generation sequencing platforms has also increased the probability of errors in genome annotations, since most genomes are annotated automatically and not subjected to any manual review. High-quality annotations of bacterial genomes are critical to understanding biological processes and enhancing these genomes has become a major task in the postgenomic era. Therefore, the reannotation of previously published genomes with manual reviewing is necessary to improve databases and supply accurate information [9]. Although numerous tools have been used to identify relevant genes, gene prediction is still imperfect. In addition, some genuine genes are missed by gene finder tools because the algorithms are directed to maintain a balance between specificity and sensitivity to avoid falsepositive predictions. Thus, the use of multiple ab initio gene finders along with BLAST searching will help to identify genes correctly and lead to more accurate annotations $[10,11]$.

Next-generation sequencing (NGS) is a cost-effective tool for the study of gene function and experimental bacterial evolution. Indeed, NGS technology was used by Luhachack and colleagues to successfully identify the function of the transcription factor $Y c j \mathrm{~W}$ as a regulator of the complex interaction between carbohydrate metabolism and H2S production in bacteria [12], and wholegenome re-sequencing in $E$. coli by Herring et al. led to the identification of mutations that conveyed a selective growth advantage during adaptation to a glycerol-based growth medium [13]. Even though the ER2566 engineering strain is widely used for the preparation of virus-like particles [14], and bacterial surface display [15], among other functions, exploring bacterial adaptive evolution of the ER2566 strain under various external pressures through NGS remains difficult and inconclusive because of limitations in the annotations of the strain [13].

In this study, we employed a series of automated annotations and combined this with manual inspections with high-throughput analyses to reannotate the ER2566 genome. As a result of our analysis, we further propose a universal reannotation pipeline for other bacterial genomes that can be undertaken with high speed and accuracy. Our reannotation eliminated 190 hypothetical genes and pseudogenes from the RefSeq annotation, and now includes an additional 237 coding sequences with definitive gene names and functions. The number of miscellaneous noncoding RNAs was also increased from 15 to 245 . Subsequently, we applied whole-genome sequencing and RNA-sequencing to assess for mutational adaptations that occur following continuous subculture pressure or overexpression burden. We detected a mutation located within the 3 ' non-coding region $(1,094,824$ position) 19-bp away from the lacI gene at the RNA level, which may be involved in the transcription and translation of genes encoding the lactose operon repressor. Our reannotation and sequencing results will provide a better understanding of some of the biological processes of the ER2566 strain, and may offer insight into future biotechnological applications in bacterial engineering. 


\section{Results \& discussions}

The process of genome reannotation combined with detailed manual reviewing encompasses the reidentification and labeling of characteristic features of a sequenced genome, and is a process that has been performed extensively for numerous organisms, including bacteria [16, 17]. Unlike the human genome, which is about $1.3 \%$ protein coding, $90 \%$ of the bacterial genome codes for proteins, with only short intergenic stretches [18]. Precise genomic annotation is thus fundamental to the further interpretation of the biochemical and physiological characteristics of organisms, to provide detailed information on protein coding sequences, pseudogenes, non-coding RNAs, repeat sequences and various other genomic data [19]. In this study, we reannotated the genome of the E. coli strain ER2566 through a reannotation pipeline, as illustrated in Fig. 1, with high speed and accuracy.

We employed a series of automated annotation tools combined with manual inspection to reannotate the ER2566 genome (Fig. S1). In our pipeline, the automation part using Prokka combined with others gene finders (GLIMMER, Zcurve and GeneMark) could finish a complete bacterial genome annotation in about 30 min. Compared with some online tools, this pipeline showed higher speed and accuracy. For example, NCBI provides a Prokaryotic Genomes Annotation Pipeline service via email, with a turn-around time of several days [7]. RAST is another web server for annotating bacterial and archaeal genomes that provides results within 1 day [20]. Some local stand-alone annotation tools, such as RATT [21], Rapid Annotation Transfer Tool, can transfer annotations from a high-quality reference to a new genome on the basis of conserved synteny. However, due to the limitation of its algorithm, RATT cannot effectively identify pseudogenes, indels, etc. Besides, numerous automated tools have been developed for genome annotation, including Mypro [22], MAKER [23], BlastLKOLA [24] and so on. To avoid false-positive predictions, the algorithms of these annotation tools are designed to balance specificity and sensitivity of their results [25]. In contrast, combination of multiple ab initio gene finders combined with BLAST searching and manual inspection will help to confirm identified genes and generate more accurate annotations.

\section{Improvement in coding sequences (CDS)}

For the systematic reannotation of the CDS, the prediction and identification of coding genes occurred in two stages (Fig. S2). In the first stage, Prodigal software was used to predict a total of 4180 CDSs on the complete ER2566 genome deposited in GenBank (accession number NC_CP014268.2). Using sequence alignment to the Swiss-Prot database [26] by Blastp [27], with a threshold e-value of $<10^{-6}$, all CDSs were annotated to provide accurate information regarding the sequences and functions of the enrolled proteins. A total of 4023 (96.2\%) of the 4180 CDSs were annotated as protein-coding genes, with the remaining CDSs (136 CDSs; 3.3\%) marked as hypothetical genes, with no registration in the SwissProt database, and 21 CDSs $(0.5 \%)$ marked as pseudogenes by manual inspection. To improve upon this prediction, three other well-established gene findersGLIMMER, Zcurve and GeneMark-were independently

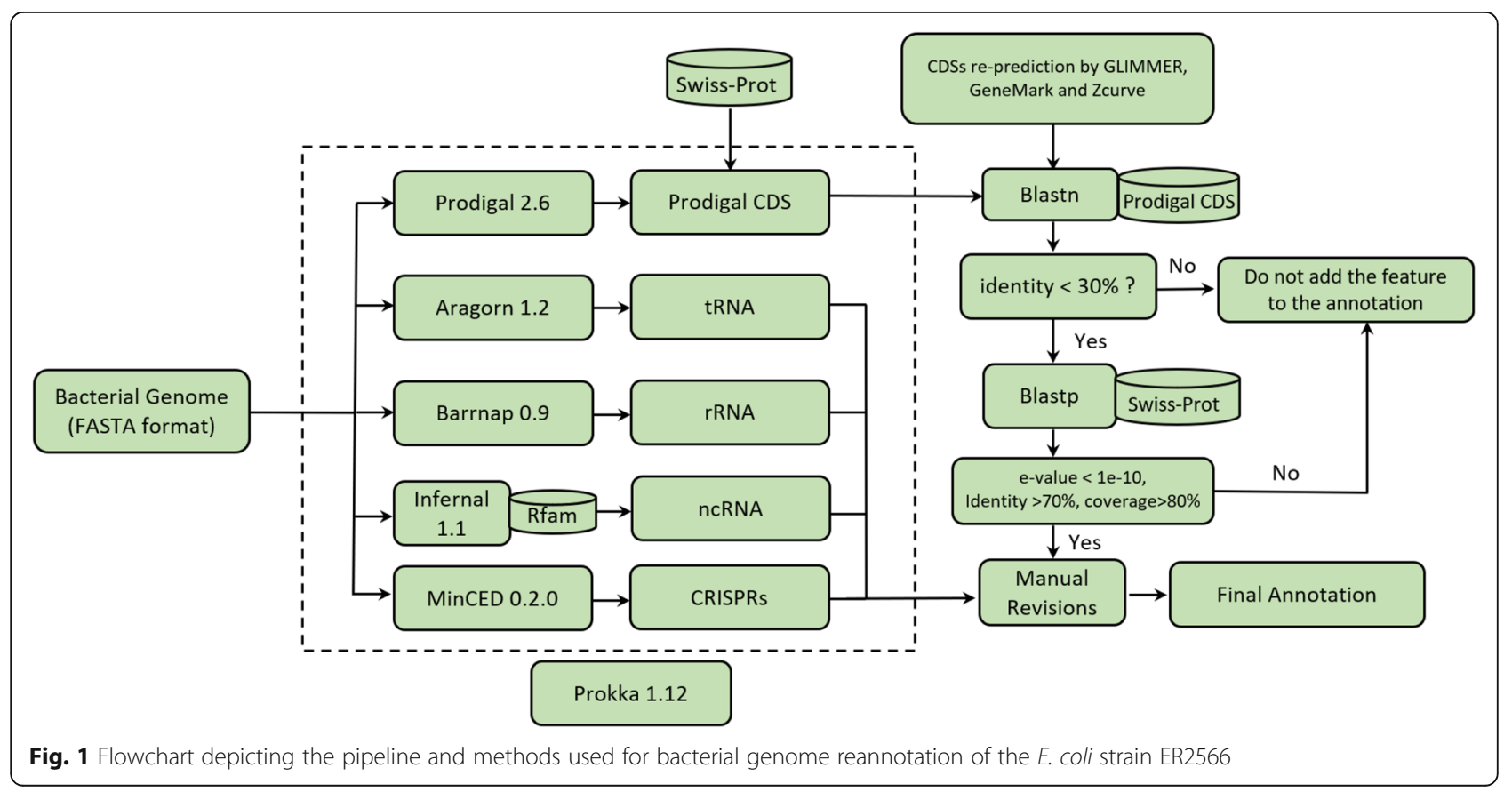


used, identifying 4231, 4287, and 4213 CDSs, respectively. These putative CDS sets were subsequently filtered by Blastn against the first Prodigal-predicted CDS set. Overall, an additional 428 CDSs were found: 194 CDSs were identified using GLIMMER, 123 using GeneMark, and 201 using ZCURVE. These additional 428 CDSs were then searched against the Swiss-Prot database by Blastp with a stricter threshold e-value $<10^{-10}$, coverage $>80 \%$, and an identity $>70 \%$. This filtered out 402 of these additional CDSs, resulting in an additional 43 genes. This led to a total of 4066 protein-coding CDSs $(4023+43)$ included in the reannotation of the ER2566 genome, along with $136 \mathrm{CDSs}$ for hypothetical genes.

Due to trimming or splitting, genes with real function can often be incorrectly assigned as pseudogenes through protein homology alignment. In our reannotation, we manually reviewed and analyzed 194 pseudogenes from the RefSeq database annotation. In total, 123 of the 194 pseudogenes were directly identified as coding genes and are now found in the reannotated list. While the remaining pseudogenes without any function had been retained. These newly identified protein-coding genes included 34 mobile genetic elements that encode transposases and had been considered to be important in evolution as a common type of genetic change. For instance, a genomic positive strand region $(3,296,328-3$, $297,025 \mathrm{bp}$ ), previously annotated as a pseudogene without function, was identified to harbor two genes, insA and insB, which are homologues of the insertion element protein, IS1, and related to DNA binding and transposase activity (Fig. 2a) [28]. In addition, two annotated pseudogenes (C2566-RS05300 and C2566_RS05310) and one hypothetical protein (C2566_RS22600) in the RefSeq annotation (range 1,088,980-1,094,720) were reannotated as three new genes (lacZ1, lacZ2, and ECBD_2906, respectively), and one related pseudogene, C2566 RS05305, was removed; these changes are consistent with previous results [5] (Fig. 2b). These three new genes are flanked by the lactose permease gene lacY upstream and the lactose operon repressor gene lacI downstream, both of which are essential to the lac operon system. This reannotation had uncovered genes related to the transcription and translation of genes encoding the lactose operon repressor in ER2566 strain.

In comparing to RefSeq annotation, a total of 190 protein-coding CDSs were removed, as they were identified as either hypothetical proteins or pseudogenes, with most of them having no assigned function (Additional file 1). Meanwhile, 237 new CDSs were added (Additional file 2). The complete reannotation list can be found in Additional file 3. ER2566 strain is a BL21/K12 hybrid strain, where about $6 \%$ sequence of $\mathrm{K}-12$ strain replaces about 7\% sequence in BL21(DE3) genome. The genome alignment of BL21 and ER2566 demonstrates a high degree of consistency [29] (Fig. 3). The recent version of BL21(DE3) annotation contains 4197

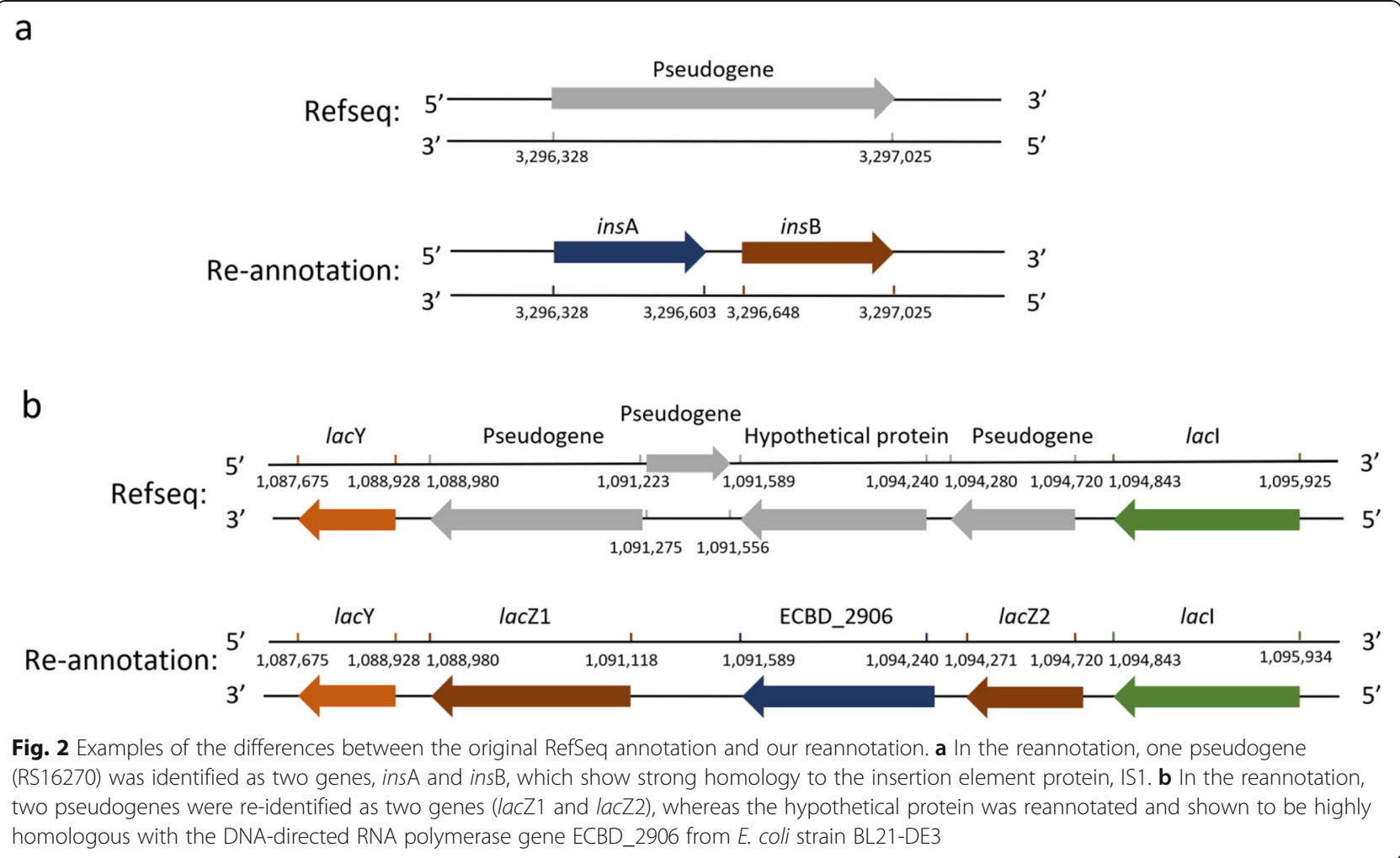




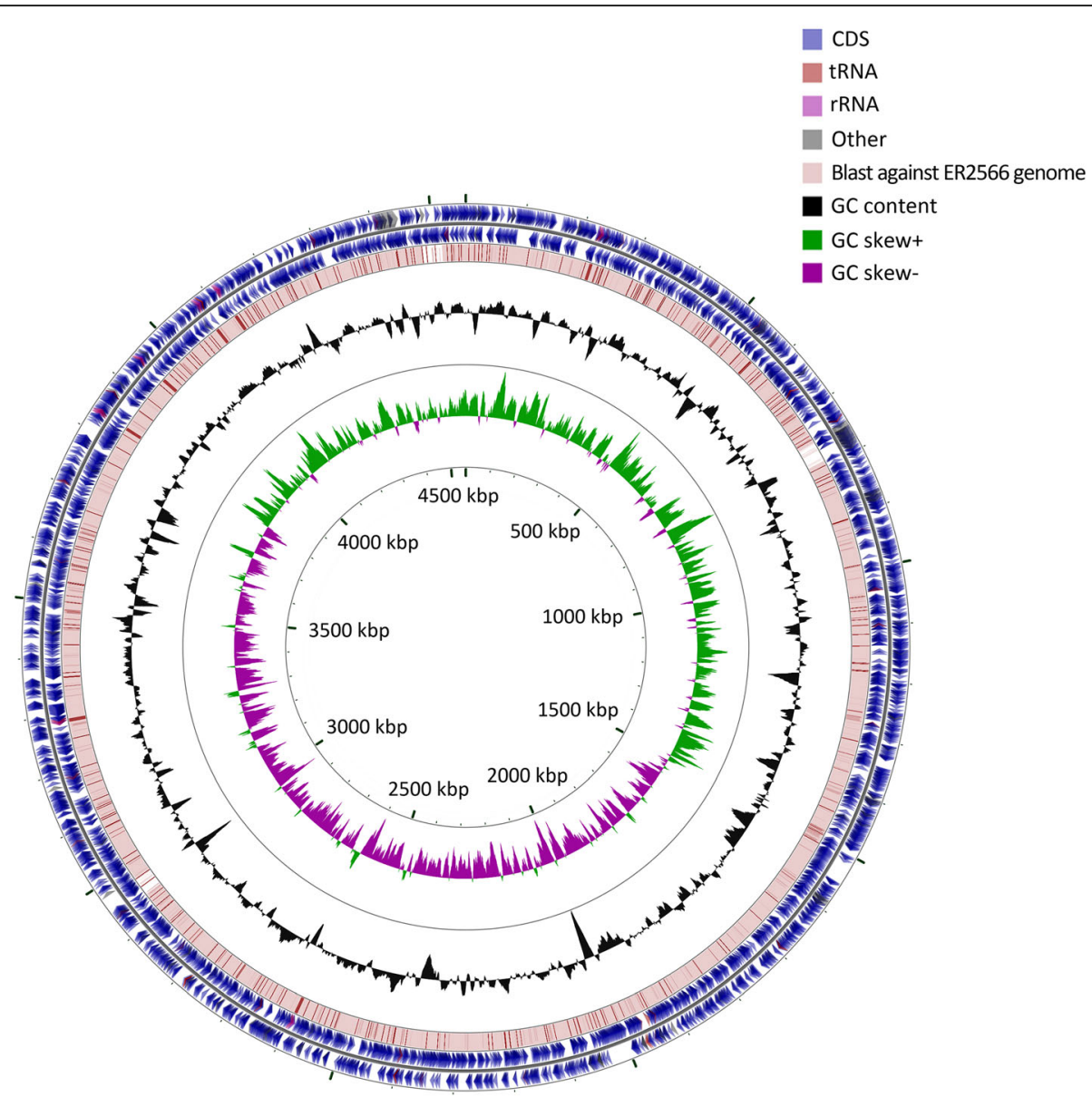

Fig. 3 Comparison between BL21(DE3) genome and ER2566 genome. Viewing from outside to inside rings, the outermost two rings, respectively representing plus-strand and minus-strand, show features extracted from the BL21(DE3) genome GenBank file (GenBank: CP001509.3); the next ring shows the positions of BLAST hits between the BL21(DE3) genome and the ER2566 genome detected by Blastn. The height of each line in the third ring showing BLAST results is proportional to the percent identity of the hit, and overlapping hits renders as darker lines. The next two rings show GC content and GC skew

Table 1 Overview of the differences between the original annotation, the reannotation and BL21(DE3) annotation

\begin{tabular}{|c|c|c|c|}
\hline & Original annotation (NZ_CP014268.2) & Reannotation & BL21(DE3) \\
\hline Genome length & $4,478,958 \mathrm{bp}$ & & $4,558,953 \mathrm{bp}$ \\
\hline Plasmids & None & & \\
\hline $\mathrm{G}+\mathrm{C} \%$ & $50.81 \%$ & & $50.83 \%$ \\
\hline Genes (total) & 4364 & 4627 & 4440 \\
\hline Protein_coding genes & 4054 & 4202 & 4197 \\
\hline Pseudo Genes & 194 & 71 & 70 \\
\hline tRNAs & 85 & 87 & 85 \\
\hline rRNAs & 22 & 22 & 22 \\
\hline Miscellaneous RNAs ${ }^{\mathrm{a}}$ & 9 & 245 & 66 \\
\hline Backbone genes & $\begin{array}{l}4170 \text { ( } 4054 \text { protein-coding genes,85 } \\
\text { tRNA genes,22rRNAs and } 9 \text { misc. RNAs) }\end{array}$ & $\begin{array}{l}4556 \text { ( } 4202 \text { protein-coding } \\
\text { genes,87 tRNA genes,22rRNAs } \\
\text { and } 245 \text { misc. RNAs) }\end{array}$ & $\begin{array}{l}4370 \text { ( } 4197 \text { protein-coding } \\
\text { genes, } 85 \text { tRNA genes,22rRNAs } \\
\text { and } 66 \text { misc. RNAs) }\end{array}$ \\
\hline
\end{tabular}

\footnotetext{
a: The concept of miscellaneous RNA includes ncRNA, tmRNA and all other ncRNAs
} 
CDSs, in which 3873 (92.3\%) CDSs were identified in ER2566 annotation as identical gene symbols or alias as well. The unidentical 7.7\% CDSs annotated in BL21 genome as compared to ER2566 is comprised of 7\% sequence corresponding to the hybrid part in ER2566 and other CDSs that does not have an official gene name (Additional file 4). Overall, we determined 4197 proteincoding genes for the ER2566 genome, including 136 CDSs labeled as hypothetical proteins and 4061 CDSs, which 3873 CDSs identical to BL21(DE3) that account for about 99\% of total 3903 CDSs (4197*93\%) equivalent to BL21(DE3) CDSs within ER2566 genome. This reannotation effectively eliminated the possibility of false interpretations introduced by the original annotation and provides a more integral view of the regulatory networks in ER2566 strain (Table 1).

Integrated proteogenomics search database (iPtgxDB) is widely used to provide protein expression evidence and could confirm the validity of the annotation, which was used to identify the short protein-coding genes that have numerous functions [30]. Thus, the combination of transcriptome data and reannotation results was used to generate an integrated proteomics database, which provided an important optimal basis for genome-scale regulatory or metabolic predictions and comprehensive exploration on the genome information and underlying gene functions (Additional file 5).

\section{Miscellaneous RNAs improvement}

Miscellaneous RNAs, such as transfer RNA (tRNA), ribosomal RNA (rRNA), and other non-coding RNAs (ncRNA), play pivotal biological roles in cellular activity. To date, about 119 RNAs molecules in the E. coli ER2566 strain have been identified, including 85 tRNAs, 22 rRNAs, and 12 ncRNAs [6]. However, almost all of these ncRNAs are missing from the original RefSeq annotation. We used Aragorn 1.2, Barrnap 0.9, and infernal 1.1 ncRNA finders independently to predict genes coding for tRNAs, rRNAs, and ncRNAs. Compared with the auto-annotation, 2 tRNAs and 230 ncRNAs are new, with most having functions in translation, DNA replication, and expression regulation (Additional file 6). In addition, most of the ncRNAs functions have been verified experimentally. For instance, about 94 of the nucleoid-associated ncRNAs molecules play key functions in DNA-RNA interactions [31]. Meanwhile, fragments per kilobase of transcript per million mapped reads (FPKM) value was used to quantify the transcription level of the newly added ncRNAs and to analyze the transcriptomics data of ER2566 under different induction conditions (Additional file 7). Quantitative analysis indicates that $85 \%(208 / 245)$ of newly added ncRNAs are detectable, while the other $15 \%(37 / 245)$ ncRNAs are undetectable possibly due to some specific functions requiring certain conditions. In summary, our reannotation introduced 230 new ncRNAs and 2 new tRNAs, with an overall tally of genes encoding for 87 tRNAs, 22 rRNAs and 245 ncRNAs.

\section{Variant calling of whole-genome re-sequencing under consecutive subculture}

Genomic variations in bacterial species usually reflect an evolutionary response that occurs under various external-usually unfavorable-environmental stressors. Thus, we next performed variant calling to identify any nucleotide-level differences (i.e., single nucleotide polymorphisms (SNPs), insertions and deletions (indels), and/or structural variations) in the ER2566 strain. There are two approaches for variant calling: by mapping reads against the reference genome directly or by assembling a de novo genome to compare against a reference genome. In most cases, mapping reads produces a better resolution for SNPs and indels than genome assembly, whereas the latter is optimal for identifying structural variants and regions with high divergence. Here, we used both methods to interrogate the ER2566 genome (Fig. 4).

First, we re-sequenced the whole genome of the ER2566 strain grown in our laboratory under consecutive subculturing. Two different-sized insert libraries $(500 \mathrm{bp}, 2000$ bp) were built, and a total of 10.0 million paired-end reads of $90 \mathrm{bp}$ in length were generated using an Illumina HiSeq 2000. The raw reads were mapped to the $\mathrm{C} 2566$ reference genome (NC_CP014268.2) with a good coverage depth (> 100-times). No SNP or indel was detected. A pipeline optimized for longer assembly was designed to accomplish the re-sequencing of our ER2566 strain. Various de novo assembly softwares were used to construct a confident and long $(4,469,460-\mathrm{bp})$ scaffold, and assembly results for each step were assessed by alignment of final sequences back to the reference genome (Fig. S3). One inversion was found (Fig. S3c), which turned out to be a genome assembly issue caused by high repetition region and was corrected by subsequent Sanger sequencing. The technical difference between short-read sequencing and single molecule long-read sequencing may result in the generation of inverted region. Nevertheless, our pipeline, by the combination of read mapping, de novo assembly and Sanger confirmation, generated an intact ER2566 genome in our practice. The resequencing of ER2566 also suggested that the continuous subculture of E. coli ER2566 strain in our lab did not cause mutation in the genomic sequence.

\section{Mutation detections by RNA-sequencing under overexpression}

RNA-seq is widely used in quantitative gene expression studies for the identification of non-annotated transcripts and polymorphisms, and for RNA editing in 


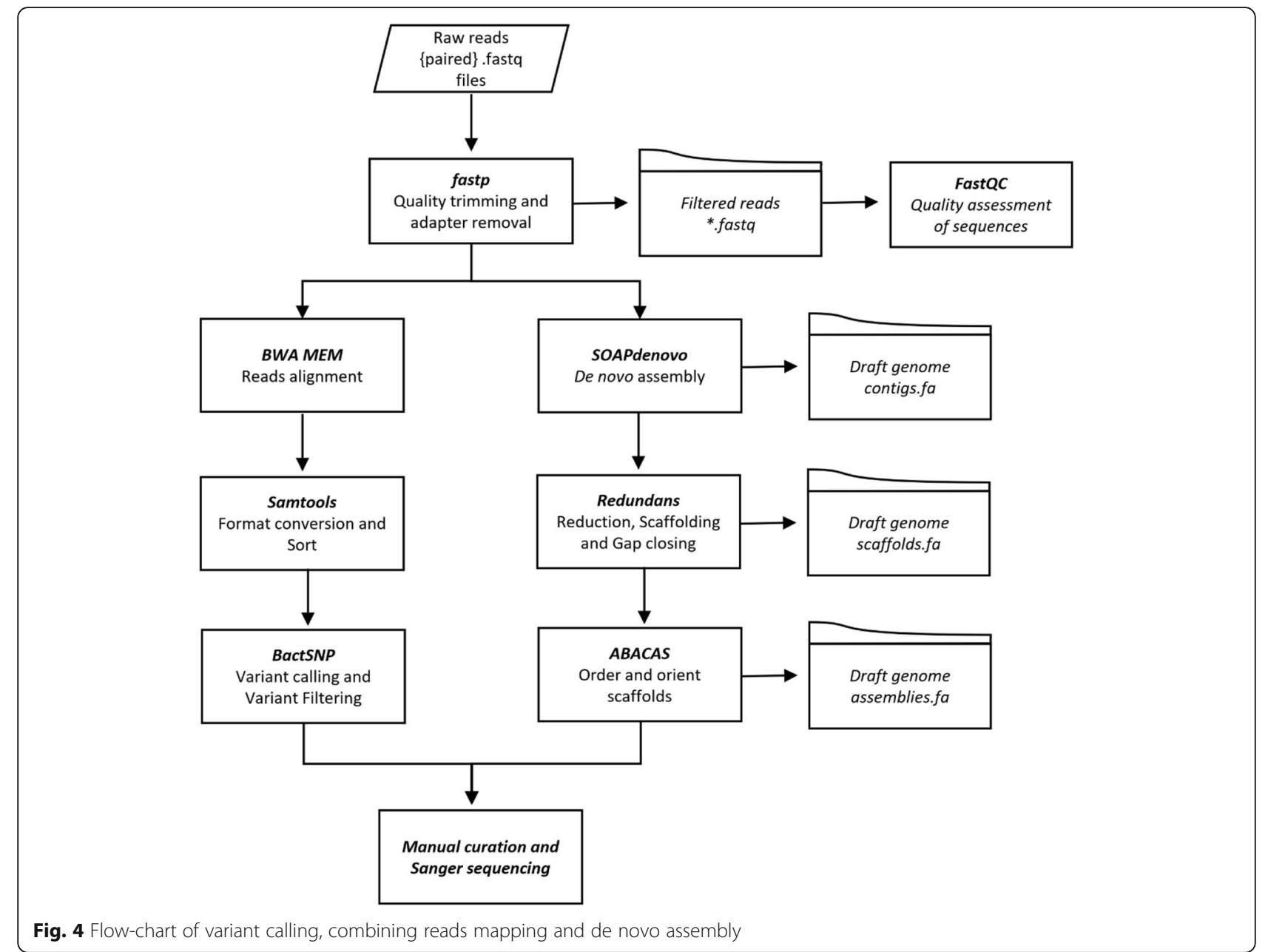

transcribed regions. Thus, to identify any variations in the ER2566 genome due to overexpression pressure, we used RNA-seq to analyze the transcriptomes of the ER2566 strain growing at $37^{\circ} \mathrm{C}$ without plasmids (B37, three replicates) or overexpressing human papillomavirus 16 type capsid protein L1 via plasmid-based inducible expression (Y37, three replicates) (Fig. 5a). From a total of 75.4 million 125 -bp paired-end reads, 73.9 million reads (98\%) were mapped to the reference genome (NC_CP014268.2) in B37 (control) samples. Yet, for the Y37 (overexpressed) samples, among the 76. 0 million reads, only 29.6 million (39\%) reads mapped to the reference genome, which was significantly lower than that for the B37 samples. The cause of lower mapping rates in Y37 samples was due to the large number of mRNAs transcribed by the engineered plasmids which is not related to genome sequence (Table 2).

The variant detection analysis using BactSNP revealed one mutation in the Y37 samples $(1,094,824$ position; Fig. 5b), located in the non-coding region 19-bp downstream from the lacI gene. Interestingly, lacI is the highest transcribed gene in the Y37 samples by comparing the FPKM for all genes (Additional file 8). The analysis of transcriptome data indicated a mutation of $\mathrm{C}$ to $\mathrm{T}$ substitution at position 109,824 in the three replicates of Y37 samples, as confirmed by nearly $100 \%$ mutation rate in all observed reads $(910,914$ Out 911,345 reads). Furthermore, the mutation was found in three replicates of B37 samples as well, with a mutation rate up to $85 \%$ (655 Out 773 reads). Surprisingly, such mutation could not be detected in the bacterial genome by Sanger sequencing (Fig. 5c). The discrepancy between transcribed RNA and genome sequence may arise from the modification during RNA transcription instead of a sequencing error. The presumption is supported by the mutation position being located in a high-efficiency RNA methylation site, which is often accompanied with spontaneous deamination of 5-methylcytosine and consequently producing thymine in aqueous solution [32]. It is interesting to clarify whether the overexpression of lacI could lead to the increase of RNA methylation rate. The function of this site will be further investigated in our future work, using a combination of gene editing, RNA methylation analysis, and other relevant techniques. 


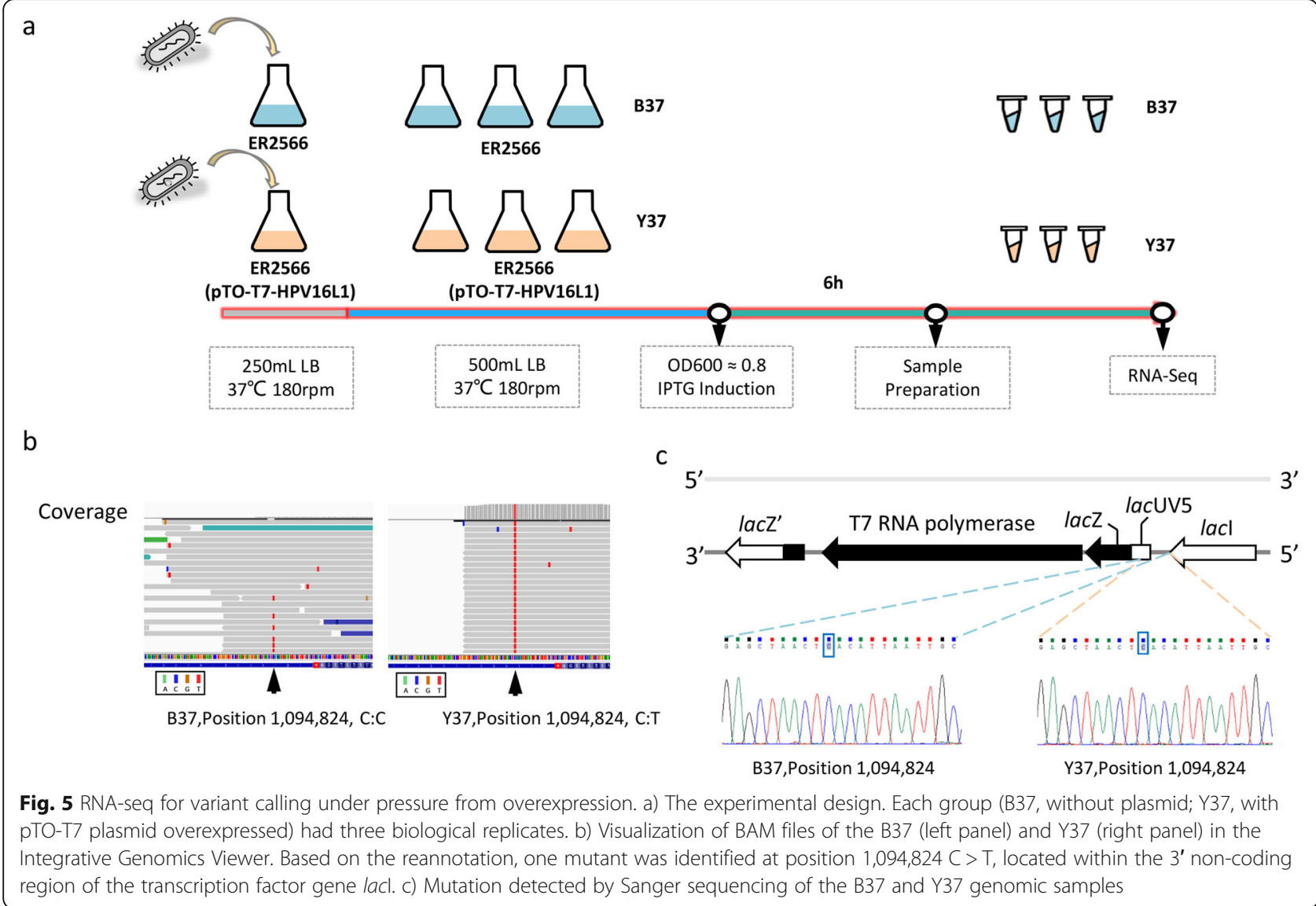

\section{Conclusions}

Here, we employed a series of automated annotation tools along with manual inspection to reannotate the ER2566 genome. The major updates include the noteworthy correction of all protein-coding genes, the exclusion of 190 CDSs from the Refseq annotation, and the addition of 237 new CDSs with definitive name or putative function. Moreover, there is an increase in the number of miscellaneous RNAs from 15 to 245. These new additions will help to provide a more informative profile of the ER2566 genome and provide a better base for exploring the molecular mechanisms of stress in response to changes in the bacterial cellular milieu. Nevertheless, this reannotation still has further room for improvement, with the continuing advancement of the algorithm, the accumulation of next-generation sequence data and proteomics data. We also carried out whole-genome sequencing and RNA-seq to detect sequence variants under different conditions of external pressure, and detected one mutation within the noncoding region of the lacI gene. However, this mutation was not detected at the genomic level by Sanger

Table 2 Statistical analysis of RNA-seq data

\begin{tabular}{llllll}
\hline Sample & Run & Raw sequences reads & Unidentified reads $^{\mathrm{a}}$ & HPV16L1 reads $^{\mathrm{b}}$ & E. coli reads $^{\mathrm{b}}$ \\
\hline B37 & 1 & $22,781,394(100 \%)$ & $250,596(1 \%)$ & $0(0 \%)$ & $22,334,273(99 \%)$ \\
& 2 & $22,763,848(100 \%)$ & $318,694(1 \%)$ & $0(0 \%)$ & $22,312,577(99 \%)$ \\
Y37 & 3 & $29,884,996(100 \%)$ & $597,700(2 \%)$ & $0(0 \%)$ & $29,276,302(98 \%)$ \\
& 1 & $28,292,578(100 \%)$ & $4,526,812(16 \%)$ & $12,731,660(45 \%)$ & $11,034,106(39 \%)$ \\
& 2 & $25,214,878(100 \%)$ & $4,286,528(17 \%)$ & $11,346,696(45 \%)$ & $9,581,654(38 \%)$ \\
& 3 & $22,521,416(100 \%)$ & $3,378,212(15 \%)$ & $10,134,638(45 \%)$ & $9,008,566(40 \%)$ \\
\hline
\end{tabular}

${ }^{a}$ : Three biological replicates of each samples were analyzed. Unidentified RNA-seq reads could include unidentified nucleotides (Ns), short reads, low quality reads, unaligned recombinant protein reads and a larger number of mRNA from plasmids. ${ }^{b}$ : HPV16L1 reads and E. coli reads respectively represent Human papillomavirus and Escherichia coli organism reads 
sequencing, which may indicate that this is an RNA modification related to the biological strain of overexpression pressure in ER2566.

The ER2566 strain is used widely within the scientific community, and our reannotation not only improved the characterization of the strain but uncovered a key site that might be involved in the transcription and translation of genes encoding the lactose operon repressor. Our reannotation pipeline with high speed and accuracy could thus be extrapolated for the reannotation of other bacterial genomes to provide a better understanding of gene function under external burden and provide more clues to engineer bacteria for biotechnological applications.

\section{Methods}

Bacterial strains, plasmids, and culture conditions

The E. coli B Strain ER2566 was purchased from New England Biolabs (NEB). Cells were grown at $37^{\circ} \mathrm{C}$ with sharking at $180 \mathrm{rpm}$ in Luria-Bertani (LB) broth $(5 \mathrm{~g} / \mathrm{L}$ $\mathrm{NaCl}, 10 \mathrm{~g} / \mathrm{L}$ tryptone, $5 \mathrm{~g} / \mathrm{L}$ yeast extract, $\mathrm{pH} 7.0$ ) under the pressure of continual passaging.

The gene encoding human papillomavirus 16 type L1 (HPV16L1) was cloned into the pTO-T7 expression vector. E. coli ER2566 with the pTO-T7-HPV16L1 vector was cultured in $250 \mathrm{~mL}$ LB broth containing $20 \mu \mathrm{g} / \mathrm{mL}$ kanamycin at $37^{\circ} \mathrm{C}$ with shaking at $180 \mathrm{rpm}$. Upon reaching an $\mathrm{OD}_{600}$ of $1.0,5 \mathrm{~mL}$ of culture was transferred into a flask containing $500 \mathrm{~mL} \mathrm{LB}$ and $20 \mu \mathrm{g} / \mathrm{mL}$ kanamycin, and incubated at $37^{\circ} \mathrm{C}$ with shaking at 180 $\mathrm{rpm}$. At $\mathrm{OD}_{600}$ of 0.8 , the culture was induced with a final concentration of $0.1 \mathrm{mM} / \mathrm{L}$ isopropyl- $\beta$-d-thiogalactopyranoside (IPTG) and incubated for $6 \mathrm{~h}$ at $37^{\circ} \mathrm{C}$ with shaking at $180 \mathrm{rpm}$.

\section{DNA extraction and sequencing}

Genomic DNA was extracted using a cetyltrimethylammonium bromide (CTAB)-based protocol [33]. Total genomic DNA concentration and quality were determined using a NanoDrop2000 Spectrophotometer (Thermo Fisher Scientific). DNA libraries for Illumina sequencing were constructed according to the manufacturer's specifications (Thermo Fisher Scientific). After DNA library construction, sequencing was performed by a commercial service (Beijing Genomic Institute, Beijing, China) on an Illumina HiSeq2000 platform with 90-bp paired-end reads. Finally, 10.7 million raw reads were obtained for subsequent analyses.

\section{RNA extraction and sequencing}

Cells were harvested by centrifugation at $7000 \mathrm{rpm}$ for $10 \mathrm{~min}$ at room temperature and total RNA was extracted using the MasterPure RNA Purification Kit, according to the manufacturer's protocol (Lucigen). DNase was added to reduce the chance of genomic DNA contamination. Total RNA was extracted in $50 \mu \mathrm{L}$ RNasefree DEPC-treated water. RNA concentration was measured using an RNA Assay Kit in a Qubit 2.0 Fluorometer. A total of $3 \mu \mathrm{g}$ RNA per sample was used as the input material for RNA sample preparations. Libraries of RNA-seq template were constructed using the NEBNext Ultra RNA Library Prep Kit for Illumina (NEB, USA), following the manufacturer's recommendations. Sequencing was performed on an Illumina HiSeq 2500 platform and 125-bp paired-end reads were generated (Novogene, Beijing, China).

\section{Genome reannotation of the ER2566 strain}

The ER2566 strain (NC_CP014268.2) whole genome was downloaded from the NCBI Reference Sequence Database as an input file for Prokka, a widely used annotation software that annotates a bacterial genome in about 10 min on a laptop [34]. To attain a rich and reliable annotation, we coordinated a suite of existing tools, including Prodigal 2.6 [35], Aragorn 1.2 [36], Barrnap 0.9 (https:// github.com/tseemann/barrnap), Infernal 1.1 [37] and MinCED 0.2.0 [38] for our reannotation pipeline to predict, respectively, coding sequences (CDS), ribosomal RNA genes (rRNA), transfer RNA genes (tRNA), noncoding RNA genes (ncRNA) and clustered regularly interspaced short palindromic repeats (CRISPRs). Subsequently, various gene finders (GLIMMER 2.03 [39], GeneMark [40] and ZCURVE [41]) were used to further confirm the coding sequences.

\section{Variant calling of whole-genome sequencing by reads mapping}

The quality of the raw reads were determined using FastQC [42], and appropriately truncated and filtered using fastp [43] to remove low-quality bases and Illumina adapter contamination with default parameters. The clean reads were then mapped against the C2566 reference genome using bwa-mem [44] with standard settings, and sorted by location as bam files. Bam files were converted to sam files using Samtools [45]. BactSNP was used to remove duplications and to detect variant calling [46].

\section{Variant calling of whole-genome sequencing by genome assembly}

Draft contigs were created using SPAdes [47], with optimized parameters. The assembled draft contigs and sequencing libraries were used as input into Redundans [48] and scaffold genome assembly was performed with the recommended parameters. This resulted in fewer fragments, longer sequences and fewer gaps, as compared with using the input contigs. Scaffolds were then further improved using ABACAS [49], which rapidly 
aligned, ordered and orientated the scaffolds based on the following user-provided references: perl abacas.pl $-r$ reference.fa $-\mathrm{q}$ scaffolds.fa $-\mathrm{p}$ nucmer. Finally, the assemblies were used to identify the mutants or indels against the reference genome through the program Harvest [50].

\section{Supplementary information}

Supplementary information accompanies this paper at https://doi.org/10 1186/s12864-020-06818-1.

Additional file 1: Table S1. The list of the ruled-out genes in the reannotation.

Additional file 2: Table S2. The list of newly added protein-coding in the reannotation.

Additional file 3: Table S3. The list of complete CDSs in the reannotation.

Additional file 4: Table S4. Comparison with BL21(DE3) and ER2566 annotation.

Additional file 5. An integrated proteogenomics search database of ER2566 strain.

Additional file 6: Table S5. The list of newly added miscellaneous ncRNAs in the reannotation.

Additional file 7: Table S6. The transcription level of the newly added ncRNAs.

Additional file 8: Table S7. The top 50 the highly expressed genes.

Additional file 9. The re-sequenced ER2566 genome. (FASTA $4436 \mathrm{~kb}$ )

Additional file 10. The reannotation of ER2566 genome.

Additional file 11: Figure S1. The analysis pipeline for genomic reannotation.

Additional file 12: Figure S2. The workflow of CDSs reannotation.

Additional file 13: Figure S3. Pairwise alignment and visualization. Assembly results for each step are assessed by alignment of final sequences back onto the reference genome. The visualization results are quickly achieved by Nucmer.

\section{Abbreviations}

BLAST: Basic local alignment search tool; CDS: Coding sequence; IPTG: Isopropyl- $\beta$-d-thiogalactopyranoside; NGS: Next-generation sequencing; ORF: Open reading frame; PGAP: Prokaryotic genome annotation pipeline; RNA-seq: RNA sequencing; SNP: Single nucleotide polymorphism; WGS: Whole-genome sequencing; FPKM: Fragments per kilobase of transcript per million mapped reads

\section{Acknowledgements}

We thank Mrs. Rebecca Jackson for polishing and revising of the final manuscript.

\section{Authors' contributions}

S.L. conceived the study. L.Z., K.W., T.C. and Y.M. performed DNA-library preparation, whole-genome sequencing and RNA-seq. L.Z., H.Y., K.W., J.L., L.L., Y.L. and H.Y. performed SNP analysis, RNA-seq analysis and reannotation. L.Z., H.Y., Z.K., Q.Z., Y.W., Y.G., H.Y., S.L. and N.X. analyzed data. L.Z., H.Y., Y.G., and S.L. wrote the manuscript. All authors have read and approved the final manuscript.

\section{Funding}

This work was supported by the Chinese government: the National Natural Science Foundation of China (grant no. U1705283, 31670935, 81971932), the Natural Science Foundation of Fujian Province (Grant 2017 J07005), and the New drug Invention project (grant no.2018ZX09738-008). The funders had no role in study design, data collection and analysis, decision to publish, or preparation of the manuscript.

\section{Availability of data and materials}

The genomic sequence of the E. coli B strain ER2566 was downloaded in FASTA format from the NCBI-Microbial Genome Database (NZ_CP014268.2) and was annotated by Prokka using multiple annotation tools and manual review. Whole-genome re-sequencing and RNA-seq data in this study were respectively deposited in the NIH Sequence Read Archive (www.ncbi.nlm.nih. gov/sra/). The raw reads of whole-genome re-sequencing for different insert sizes:ER2566_500-SRR10828732 (https://trace.ncbi.nlm.nih.gov/Traces/sra/ ?run=SRR10828732), ER2566 2000-SRR10828731 (https://trace.ncbi.nlm.nih. gov/Traces/sra/?run=SRR10828731), RNA-Seq raw data:BB371SRR10828730(https://trace.ncbi.nlm.nih.gov/Traces/sra/?run=SRR10828730), BB372-SRR10828729(https://trace.ncbi.nlm.nih.gov/Traces/sra/?run=SRR1082 8729), BB373-SRR108287828(https://trace.ncbi.nlm.nih.gov/Traces/sra/?run= SRR10828728), YY371-SRR108287827(https://trace.ncbi.nlm.nih.gov/Traces/sra/ ?run=SRR10828727), YY372-SRR108287826(https://trace.ncbi.nlm.nih.gov/ Traces/sra/?run=SRR10828726), YY373-SRR108287825(https://trace.ncbi.nlm. nih.gov/Traces/sra/?run=SRR10828725). The assembly and reannotation of the ER2566 genome had been introduced a supplementary file (Additional files 9 and 10).

Ethics approval and consent to participate

Not applicable.

\section{Consent for publication}

Not applicable.

\section{Competing interests}

The authors declare that they have no competing interests.

Received: 9 January 2020 Accepted: 10 June 2020

Published online: 16 June 2020

\section{References}

1. Sezonov G, Joseleau-Petit D, D'Ari R. Escherichia coli physiology in LuriaBertani broth. J Bacteriol. 2007;189(23):8746-9.

2. Shiloach J, Fass R. Growing E-coli to high cell density - a historical perspective on method development. Biotechnol Adv. 2005;23(5):345-57.

3. Rosano GL, Ceccarelli EA. Recombinant protein expression in Escherichia coli: advances and challenges. Front Microbiol. 2014;5.

4. Correa A, Oppezzo P. Overcoming the solubility problem in E. coli: available approaches for recombinant protein production. Methods Mol Biol. 2015; 1258:27-44.

5. Fomenkov A, Sun Z, Dila DK, Anton BP, Roberts RJ, Raleigh EA. EcoBLMcrX, a classical modification-dependent restriction enzyme in Escherichia coli B: characterization in vivo and in vitro with a new approach to cleavage site determination. PLoS One. 2017:12(6):e0179853.

6. Anton BP, Fomenkov A, Raleigh EA, Berkmen M. Complete Genome Sequence of the Engineered Escherichia coli SHuffle Strains and Their WildType Parents. Genome Announc. 2016;4:2.

7. Tatusova T, DiCuccio M, Badretdin A, Chetvernin V, Nawrocki EP, Zaslavsky L, Lomsadze A, Pruitt KD, Borodovsky M, Ostell J. NCBI prokaryotic genome annotation pipeline. Nucleic Acids Res. 2016;44(14):6614-24.

8. Goodwin S, McPherson JD, McCombie WR. Coming of age: ten years of next-generation sequencing technologies. Nat Rev Genet. 2016;17(6):333-51.

9. Ouzounis CA, Karp PD. The past, present and future of genome-wide reannotation. Genome Biol. 2002;3(2):COMMENT2001.

10. Luo CW, Hu GQ, Zhu HQ. Genome reannotation of Escherichia coli CFT073 with new insights into virulence. BMC Genomics. 2009;10.

11. Warren AS, Archuleta J, Feng WC, Setubal JC. Missing genes in the annotation of prokaryotic genomes. Bmc Bioinformatics. 2010;11.

12. Luhachack L, Rasouly A, Shamovsky I, Nudler E. Transcription factor YcjW controls the emergency H2S production in E. coli. Nat Commun. 2019;10(1): 2868.

13. Herring CD, Raghunathan A, Honisch C, Patel T, Applebee MK, Joyce AR, Albert TJ, Blattner FR, van den Boom D, Cantor CR, et al. Comparative genome sequencing of Escherichia coli allows observation of bacterial evolution on a laboratory timescale. Nat Genet. 2006;38(12):1406-12.

14. Zhang X, Wei M, Pan H, Lin Z, Wang K, Weng Z, Zhu Y, Xin L, Zhang J, Li S, et al. Robust manufacturing and comprehensive characterization of recombinant hepatitis E virus-like particles in Hecolin((R)). Vaccine. 2014 32(32):4039-50 
15. Chen TT, Wang KH, Chi X, Zhou LZ, Li JJ, Liu LQ, Zheng QB, Wang YB, Yu H, Gu Y, et al. Construction of a bacterial surface display system based on outer membrane protein F. Microb Cell Factories. 2019;18.

16. Chowdhary N, Selvaraj A, KrishnaKumaar L, Kumar GR. Genome Wide ReAnnotation of Caldicellulosiruptor saccharolyticus with New Insights into Genes Involved in Biomass Degradation and Hydrogen Production. PLoS One. 2015;10:7.

17. Slager J, Aprianto R, Veening JW. Deep genome annotation of the opportunistic human pathogen Streptococcus pneumoniae D39. Nucleic Acids Res. 2018:46(19):9971-89.

18. Salzberg SL. Next-generation genome annotation: we still struggle to get it right. Genome Biol. 2019;20(1):92.

19. Armengaud J. Reannotation of genomes by means of proteomics data. Proteomics Biol Pt A. 2017;585:201-16.

20. Aziz RK, Bartels D, Best AA, DeJongh M, Disz T, Edwards RA, Formsma K Gerdes S, Glass EM, Kubal M, et al. The RAST server: rapid annotations using subsystems technology. BMC Genomics. 2008;9:75.

21. Otto TD, Dillon GP, Degrave WS, Berriman M: RATT: Rapid Annotation Transfer Tool. Nucleic Acids Res 2011, 39(9).

22. Liao YC, Lin HH, Sabharwal A, Haase EM, Scannapieco FA. MyPro: a seamless pipeline for automated prokaryotic genome assembly and annotation. J Microbiol Methods. 2015;113:72-4.

23. Campbell MS, Holt C, Moore B, Yandell M. Genome Annotation and Curation Using MAKER and MAKER-P. Curr Protoc Bioinformatics. 2014;48(4): 11-39.

24. Kanehisa M, Sato Y, Morishima K. BlastKOALA and GhostKOALA: KEGG tools for functional characterization of genome and Metagenome sequences. J Mol Biol. 2016;428(4):726-31.

25. Sharma V, Hiller M. Increased alignment sensitivity improves the usage of genome alignments for comparative gene annotation. Nucleic Acids Res. 2017;45(14):8369-77

26. Bairoch A, Apweiler R. The SWISS-PROT protein sequence database and its supplement TrEMBL in 2000. Nucleic Acids Res. 2000;28(1):45-8.

27. McGinnis S, Madden TL. BLAST: at the core of a powerful and diverse set of sequence analysis tools. Nucleic Acids Res. 2004;32:W20-5.

28. Badia J, Ibanez E, Sabate M, Baldoma L, Aguilar J. A rare 920-kilobase chromosomal inversion mediated by IS1 transposition causes constitutive expression of the yiaK-S operon for carbohydrate utilization in Escherichia coli. J Biol Chem. 1998;273(14):8376-81.

29. Kim S, Jeong H, Kim EY, Kim JF, Lee SY, Yoon SH. Genomic and transcriptomic landscape of Escherichia coli BL21(DE3). Nucleic Acids Res. 2017:45(9):5285-93.

30. Storz G, Wolf Yl, Ramamurthi KS. Small proteins can no longer be ignored. Annu Rev Biochem. 2014;83:753-77.

31. Qian Z, Zhurkin VB, Adhya S. DNA-RNA interactions are critical for chromosome condensation in Escherichia coli. P Natl Acad Sci USA. 2017; 114(46):12225-30.

32. Nabel CS, Manning SA, Kohli RM. The curious chemical biology of cytosine: deamination, methylation, and oxidation as modulators of genomic potential. ACS Chem Biol. 2012;7(1):20-30.

33. Sharma RC, Murphy AJ, DeWald MG, Schimke RT. A rapid procedure for isolation of RNA-free genomic DNA from mammalian cells. Biotechniques. 1993;14(2):176-8.

34. Seemann T. Prokka: rapid prokaryotic genome annotation. Bioinformatics. 2014;30(14):2068-9.

35. Hyatt D, Chen GL, LoCascio PF, Land ML, Larimer FW, Hauser LJ. Prodigal: prokaryotic gene recognition and translation initiation site identification. Bmc Bioinformatics. 2010;11:119.

36. Laslett D, Canback B. ARAGORN, a program to detect tRNA genes and tmRNA genes in nucleotide sequences. Nucleic Acids Res. 2004;32(1):11-6.

37. Nawrocki EP, Kolbe DL, Eddy SR. Infernal 1.0: inference of RNA alignments. Bioinformatics. 2009;25(10):1335-7.

38. Bland C, Ramsey TL, Sabree F, Lowe M, Brown K, Kyrpides NC, Hugenholtz P. CRISPR recognition tool (CRT): a tool for automatic detection of clustered regularly interspaced palindromic repeats. Bmc Bioinformatics. 2007;8.

39. Delcher AL, Harmon D, Kasif S, White O, Salzberg SL. Improved microbia gene identification with GLIMMER. Nucleic Acids Res. 1999;27(23):4636-41.

40. Lukashin AV, Borodovsky M. GeneMark.Hmm: new solutions for gene finding. Nucleic Acids Res. 1998;26(4):1107-15.

41. Guo FB, Zhang CT. ZCURVE_V: a new self-training system for recognizing protein-coding genes in viral and phage genomes. Bmc Bioinformatics. 2006;7.
42. Brown J, Pirrung M, McCue LA. FQC dashboard: integrates FastQC results into a web-based, interactive, and extensible FASTQ quality control tool. Bioinformatics. 2017;33(19):3137-9.

43. Chen SF, Zhou YQ, Chen YR. Gu J: fastp: an ultra-fast all-in-one FASTQ preprocessor. Bioinformatics. 2018;34(17):884-90.

44. Houtgast EJ, Sima VM, Bertels K, Al-Ars Z. Hardware acceleration of BWAMEM genomic short read mapping for longer read lengths. Comput Biol Chem. 2018;75:54-64.

45. Li H, Handsaker B, Wysoker A, Fennell T, Ruan J, Homer N, Marth G, Abecasis $G$, Durbin R. Genome project data processing S: the sequence alignment/ map format and SAMtools. Bioinformatics. 2009;25(16):2078-9.

46. Brouard JS, Schenkel F, Marete A, Bissonnette N. The GATK joint genotyping workflow is appropriate for calling variants in RNA-seq experiments. J Anim Sci Biotechnol. 2019;10:44.

47. Bankevich A, Nurk S, Antipov D, Gurevich AA, Dvorkin M, Kulikov AS, Lesin VM, Nikolenko SI, Pham S, Prjibelski AD, et al. SPAdes: a new genome assembly algorithm and its applications to single-cell sequencing. J Comput Biol. 2012;19(5):455-77.

48. Pryszcz LP, Gabaldon T. Redundans: an assembly pipeline for highly heterozygous genomes. Nucleic Acids Res. 2016;44(12):e113.

49. Assefa S, Keane TM, Otto TD, Newbold C, Berriman M. ABACAS: algorithmbased automatic contiguation of assembled sequences. Bioinformatics. 2009;25(15):1968-9.

50. Treangen TJ, Ondov BD, Koren S, Phillippy AM. The harvest suite for rapid core-genome alignment and visualization of thousands of intraspecific microbial genomes. Genome Biol. 2014;15(11):524.

\section{Publisher's Note}

Springer Nature remains neutral with regard to jurisdictional claims in published maps and institutional affiliations.

Ready to submit your research? Choose BMC and benefit from

- fast, convenient online submission

- thorough peer review by experienced researchers in your field

- rapid publication on acceptance

- support for research data, including large and complex data types

- gold Open Access which fosters wider collaboration and increased citations

- maximum visibility for your research: over $100 \mathrm{M}$ website views per year

At BMC, research is always in progress.

Learn more biomedcentral.com/submissions 